\title{
Osteoradionecrosis of jaw- a review
}

\begin{abstract}
Osteoradionecrosis (ORN) jaw is a serious complication after radiation that involves the osseous structures of jaw mostly mandible. Pathophysiology is radiation induced fibroatrophic process. The predisposing factor is mostly trauma (extraction) and sometimes spontaneous. Marx staging of ORN is used for diagnosis and treatment with surgical and oxygen therapy. The preventive measure includes avoiding predisposing factors and medical management.
\end{abstract}

Keywords: osteoradionecrosis, marx staging, medical and surgical management
Volume 6 Issue 5 - 2017

\author{
Iccha Kumar Maharjan,' Ravi Kiran Ongole,2 \\ Mehul Jaisani' \\ 'Department of Oral Medicine and Radiology, Manipal college of \\ Dental Science, Mangalore, India \\ ${ }^{2}$ Department of Oral and Maxillofacial surgery, B.P.K.I.H.S, Nepal
}

Correspondence: Iccha Kumar Maharjan, Department of Oral Medicine and Radiology, College of Dental Surgery, B.P.K.I.H.S, Dharan, Nepal,Tel 9780000000000, Fax 977252025I, Email mailingiccha@gmail.com

Received: August 26, 2016 | Published: March 20, 2017

\section{Introduction}

Osteoradionecrosis $(\mathrm{ORN})$ or post radiation osteonecrosis (PRN) is a debilitating complication after radiation therapy for the head and neck cancer that involves the osseous structures within the radiation zone. ORN is defined as an area of exposed irradiated bone tissue that fails to heal over a period of 3 months without a residual or recurrent tumor. ${ }^{1}$ ORN is a bone ischaemic necrosis caused by " $3 \mathrm{H}$ " hypovascular, hypocellular, and hypoxic tissue and tissue breakdown (i.e. cellular death and collagenolysis that exceed cellular replication and collagen synthesis) followed by a nonhealing wound (e.g. tooth extraction), in which oxygen and metabolic demand exceed supply thus inhibiting substitution of cells to complete the turnover for the maintenance of homeostasis and wound cicatrisation. ${ }^{1-3}$ The affected cells are the ones of vascular endothelium, fibroblasts which makes stroma and parenchyma cells. ${ }^{3}$ The newer concept of ORN has three phases: prefibrotic phase, organized phase and fibro atrophic phase but it disagrees with hypoxia as a persistent cause for ORN. ${ }^{4}$ Mandible is most commonly involved in 2.6 to $22 \%$ and lesser in the maxilla. ${ }^{5}$ Various predisposing factors associated are trauma (from surgical procedures), active periodontal disease or denture trauma, idiopathic or spontaneous necrosis, high-dose radiation $>65 \mathrm{Gray}$, field of radiation (volume of the mandible included in the field and proximity of maximal dosing to bone), use of implant sources too close to the bone, and combined interstitial and external beam irradiation. Marx staging and treatment of osteoradionecrosis was considered the most effective tool to approach such patients. ${ }^{2,3}$ The three stages classification proposed by Marx and Myers appears to be the most relevant:

1. Stage 1: less than $2 \mathrm{~mm}$ of exposed bone with or without pain and with radiological signs of diffuse demineralisation.

2. Stage 2: more than $2 \mathrm{~mm}$ of exposed bone.

3. Stage 3: pathological fracture, oral fistula, fistula or lesion of the inferior border of the mandible. ${ }^{6}$
Treatment modalities in stage 1 and 2 are medical management and in later advances and refractory stages not responding require hyperbaric oxygen therapy with surgical therapy and reconstruction grafts. Before radiotherapy preventive measure like oral prophylaxis, flouride application and extractions should allow as much healing time as possible; with minimal trauma at least 2 weeks, ideally 3 weeks, before initiation of radiation therapy. ${ }^{7,8}$ After radiotherapy measure to reduce osteoradionecrosis include endodontic therapy over extraction, avoid using denture after 60 Gray, use of local anaesthetics that contain no or low concentration epinephrine, atraumatic surgical procedures (if surgery is necessary); prophylactic antibiotics plus post-surgical antibiotics during the week of healing and hyperbaric oxygen before invasive procedure. ${ }^{9}$ Preventive measure ORN are taken 1 week before extraction, Pentoxyfylline $(800 \mathrm{mg} / \mathrm{d})$ and Alpha-Tocopherol (vitamin E) $1000 \mathrm{gm} / \mathrm{d}$ (Evion) for 2 months if ORN develop continue for 6 months and add Clodronate (bisphosphonates) after 3 months $1600 \mathrm{mg} /$ day 5 days a week for 6 to 12 months and if sequestrum is present give all the three drugs for 3 months before the planned sequestrectomy. Pentoxifylline (PEN, increase oxygenation) and alpha-Tocopherol (TO, antioxidant) synergistically reduces radiation induced fibrosis and is potentiation by combination with Clodronate (CLO, reduces bone destruction) also called PENTOCLO protocol for 3 to 12 months appears to be effective in ORN. First phase of protocol for treatment includes anti-inflammatory (20 $\mathrm{mg}$ of prednisone), antifungal (50 mg of fluconazole), antibiotic ( $2 \mathrm{~g}$ of amoxicillinclavulanic acid/1 $\mathrm{g}$ of ciprofloxacin) and $20 \mathrm{mg}$ omeprazole daily for 4 to 6 weeks to control local super infection in the irradiated zone followed by second phase of PENTOCLO protocol. Hyper baric oxygen therapy can also be used. ${ }^{10,11}$

\section{Conclusion}

The main goal of management is to prevent ORN by measures prior to the radiation but in diseased, prognosis of ORN depends on the stage of the diagnosis with early stage having better treatment outcome with medical management and later stages requiring surgical therapy and reconstruction with grafts. 


\section{Funding}

None.

\section{Acknowledgements}

None.

\section{Conflicts of interest}

The authors declare that there is no conflict of interest.

\section{References}

1. Marx RE. Osteoradionecrosis: a new concept of its pathophysiology. $J$ oral Maxillofac Surg. 1983;41(5):283-288.

2. Cronje FJ. A review of Marx protocols: Prevention and management of Osteoradionecrosis by combining surgery and hyperbaric oxygen therapy. SADJ. 1998;53(10):469-471.

3. Marx RE. A new concept in the treatment of osteoradionecrosis. J Oral Maxillofac Surg. 1983;41(6):351-357.

4. Lyons J, Ghazali N. Osteoradionecrosis of the jaw: current understanding of its pathology. Br J Oral Maxillofac Surg. 2008;46(8):653-660.
5. Thorn JJ, Sand Hansen H, Specht L, et al. Osteoradionecrosis of the jaws: clinical characteristics and relation to the field of irradiation. J Oral Maxillofac Surg. 2000;58(10):1088-1095.

6. Myers R, Marx RE. Use of hyperbaric oxygen in postradiation head and neck surgery. Natl Cancer Inst Monogr. 1990;90:151-157.

7. Beumer J, Harrison R, Sanders B, et al. Post radiation dental extractions: a review of the literature and a report of 72 episodes. Head Neck Surg. 1983;6(1):581-586.

8. Ferguson HW, Stevens MR. Advances in head and neck radiotherapy to the mandible. Oral Maxillofacial Surg Clin N Am. 2007; 19(4):553-563.

9. Max RE, Johnson RP, Kline SN. Prevention of osteoradionecrosis: A randomized prospective clinical trial of hyperbaric oxygen versus penicillin. J Am Dent Assoc. 1985;111(1):49-54.

10. Delanian S, Depondt, Lefaix JL. Major healing of refractory mandible osteoradionecrosis after treatment combining Pentoxyfylline and tocopherol: a phase II trial. Head Neck. 2005;27(2):114-123.

11. Robard L, Louis MY, Blanchard D, et al. Medical treatment of osteoradionecrosis of the mandible by PENTOCLO: Preliminary results: A preliminary results. Eur Annal Otorhinolaryngol Head Neck Dis. 2014;131(6):333-338. 